\title{
Spatial characterization of nanotextured surfaces by visual color imaging
}

Feidenhans'I, Nikolaj Agentoft; Murthy, Swathi; Madsen, Morten H.; Petersen, Jan C.; Taboryski, Rafael J.

\section{Published in:}

Applied Optics

Link to article, DOI:

10.1364/AO.55.009719

Publication date:

2016

Document Version

Peer reviewed version

Link back to DTU Orbit

Citation (APA):

Feidenhans'I, N. A., Murthy, S., Madsen, M. H., Petersen, J. C., \& Taboryski, R. J. (2016). Spatial characterization of nanotextured surfaces by visual color imaging. Applied Optics, 55(34), [9719]. https://doi.org/10.1364/AO.55.009719

\section{General rights}

Copyright and moral rights for the publications made accessible in the public portal are retained by the authors and/or other copyright owners and it is a condition of accessing publications that users recognise and abide by the legal requirements associated with these rights.

- Users may download and print one copy of any publication from the public portal for the purpose of private study or research.

- You may not further distribute the material or use it for any profit-making activity or commercial gain

- You may freely distribute the URL identifying the publication in the public portal

If you believe that this document breaches copyright please contact us providing details, and we will remove access to the work immediately and investigate your claim 


\title{
Spatial characterization of nanotextured surfaces by visual color imaging
}

\author{
Nikolaj A. Feidenhans’ ${ }^{1,2}$, Swathi Murthy ${ }^{3,4}$, Morten H. Madsen ${ }^{1}$, Jan C. \\ Petersen $^{1, *}$, RAfAel TABORYSKI ${ }^{2, *}$ \\ ${ }^{1}$ Danish Fundamental Metrology Ltd., Matematiktorvet 307, 2800 Kgs. Lyngby, Denmark \\ ${ }^{2}$ Department of Micro- and Nanotechnology, Technical University of Denmark, 2800 Kgs. Lyngby, Denmark \\ ${ }^{3}$ Department of Photonics Engineering, Technical University of Denmark, $2800 \mathrm{Kgs}$. Lyngby, Denmark \\ ${ }^{4}$ Inmold A/S, Gregersensvej 6H, 2630 Taastrup, Denmark \\ *Corresponding authors: jcp@dfm.dk, rata@nanotech.dtu.dk
}

Received XX Month XXXX; revised XX Month, XXXX; accepted XX Month XXXX; posted XX Month XXXX (Doc. ID XXXXX); published XX Month XXXX

\begin{abstract}
We present a method for characterization of nanostructures from the visual color of the structures, obtained with an ordinary color camera. The method provides a macroscale overview image from which micrometer sized regions can be analyzed independently, hereby revealing long-ranged spatial variations of the structures. The method is tested on injection molded polymer line gratings, and the height and filling factor determined with confidence intervals similar to more advanced imaging scatterometry setups. (C) 2015 Optical Society of America
\end{abstract}

OCIS codes: (120.0120) Instrumentation, measurement, and metrology, (120.4630) Optical inspection, (150.0150) Machine vision, (150.3040) Industrial inspection, (150.5495) Process monitoring and control s.

http://dx.doi.org/10.1364/AO.99.099999

\section{INTRODUCTION}

The development of structured functional surfaces is currently receiving increased focus, with applications such as generating color effects $[1,2]$ or altering wetting behaviors $[3,4]$. Simultaneously several groups have demonstrated the fabrication of such micro and nanostructures using large-scale production methods like injection molding [5] and roll-to-roll manufacturing [6-9]. However, with high volume production methods, characterization of the structures is becoming an increasing issue. The current workhorses for characterization of micro/nano-scale structures are atomic force microscopy (AFM) and scanning electron microscopy (SEM) [10]. AFM is excellent for evaluating structure heights and period, but the recording of each image takes many minutes and the field of view is limited $(<100 \mu \mathrm{m})$. SEM has a larger field of view, but evaluation of vertical structures is difficult and the sample size restricted by the instrument vacuum chamber. For adoption of structured functional surfaces into mass produced products, faster characterizations techniques are needed.

In the semiconductor industry, scatterometry is increasingly being employed for critical dimension metrology $[10,11]$. Scatterometry is an optical characterization technique for periodic structures, where the structure dimensions are determined by fitting the measured reflection efficiencies to a simulation database, as the reflection efficiencies depends on the structure dimensions. To obtain good fitting confidence several values are obtained for each sample, typically by including multiple diffraction orders, or by varying parameters such as the wavelength, polarization, or incident angle of the probe light [10]. For generating the simulation database, the approximate structure dimensions must be known a priori. The current approaches are mainly targeting precise measurements of the very high quality samples produced by the semiconductor industry $[11,12]$. However, for industrial polymer production single nanometer precision is not needed, as the samples cannot be expected to have perfectly well defined shapes.

We present a new method, based on scatterometry, which can determine the height, filling factor, and sidewall slope of nanoscale 1dimensional (1D) line gratings, simply by evaluating the color of the sample. The use of visual colors in scatterometry has previously been reported, but only with focus on the human ability to distinguish colors [13-15]. In the presented setup, the color is determined as the RGB values obtained with an ordinary camera, and the method is named "color scatterometry". The motivation is that such gratings can be added for quality control purposes in areas of no significance for the products functionality. The advantage over other scatterometry methods, such as spectroscopic scatterometry, is that it provides an overview image of several $\mathrm{mm}^{2}$ from which smaller areas subsequently can be analyzed independently. This is similar to imaging scatterometry [16], where hyper-spectral imaging is used to obtain the reflection spectrum for each pixel in an image. However, where hyperspectral requires around 50 images obtained at different wavelengths, color scatterometry only requires a single image acquisition, hereby providing significantly shorter evaluation times.

As we have previously shown, scatterometry can also be used for characterizing random roughness on polished surfaces [17]. However, 
due to the broad range of structural surface wavelengths, such analysis is generally not possible with spectroscopic scatterometry due to the narrow range of probe wavelengths. Instead the method of angular scatterometry is utilized, where the magnitude and frequency of the surface features determines the intensities in the scattering distribution [18]

\section{METHODS}

Polymer test samples were manufactured by polymer injection molding using a nickel shim electroformed from a silicon wafer structured with the negative pattern, which had been fabricated by semiconductor processing techniques as described in Ref [6] . Injection molding was performed on an industry grade injection molding machine (Engel Victory Tech 80/45, ENGEL AUSTRIA GmbH, Austria) using a variotherm process. The polymer used was cyclic olefin copolymer (TOPAS5013L-10, TOPAS Advanced Polymers $\mathrm{GmbH}$, Germany) mixed with $1.15 \%$ black pigment (UNS 949227, Gabriel-Chemie Group, Austria). The black pigment provides an enhanced visual appearance of the structured colors, due to a surrounding dark background, but does not affect the structural colors.

The samples consist of a single polymer disc with seven areas of 4.5 $\mathrm{x} 4.5 \mathrm{~mm}^{2}$, patterned with rectangular shaped line gratings of different periods, as shown in Figure 1a-d. The design dimensions were: height $h=210 \mathrm{~nm}$, projected area filling factor $f=0.50$, sidewall slope $\alpha=0^{\circ}$, and periods $p$ of $600,800,1000,1200,1400,2000$ and $5000 \mathrm{~nm}$. The dimensions were chosen to provide high reflection efficiencies, but not with respect to the detection sensitivity. The actual dimensions of each sample were determined by a combination of AFM (Park NX20, Park Systems, South Korea) and focused ion-beam electron microscopy (FIB SEM) (FEI Helios Nanolab 600, FEI, USA) and (Zeiss Supra VP 40, Zeiss, Germany). Two methods were employed, as none of them alone could evaluate the full range of dimensions obtained by color scatterometry. AFM is superior for measuring heights, but not well suited for sidewall slopes or filling factors, due to tip convolution. With FIB milling a trench was cut perpendicular to the grating, hereby revealing a cross section of the profile, from which the filling factor and slope was determined from SEM images. The final reference values were obtained as the mean of several grating profiles, and the $95 \%$ confidence intervals (CI) determined as: $\mu \pm t_{n-1} \cdot S E$, where $\mu$ is the mean value, $S E$ is the standard error of the mean, corrected for small sample bias by Cochran's theorem [19], and $t$ is the Student's $t$ distribution for $n$ samples ( $n=2$ to 9 , typically 4 or 5 ).

The refractive index of the polymer was determined by ellipsometry (VASE, J.A. Woollam Co., USA), on the unstructured center region of the disc. The measurements were performed at three incident angles, and the wavelength dependent complex refractive index ( $\tilde{n})$ determined by the instrument software, by fitting the Fresnel reflection coefficients to the evaluated amplitude and phase components, for each wavelength individually. For the interesting visible region $(380 \mathrm{~nm}-800 \mathrm{~nm})$ the typical value was around $\tilde{n}=1.55+i 0.005$.

The colors reflected from each sample were evaluated with a custom-built setup consisting of: a broadband LED lamp, three irises, a polarizing beamsplitter, and a RGB color camera, see Figure 2ab. The lamp provides unpolarized light, partially collimated by two irises, while the last iris blocks higher order diffractions. The polarizing beamsplitter effectively functions as a crossed polarizer and analyzer, by blocking the dominant reflection of unchanged polarization, and only transmits light that is rotated from $s$ - to $p$-polarization. This polarization setup provides significantly larger color changes with respect to the grating dimensions, and outperforms typical scatterometry setups that use non-polarizing 50/50 beamsplitters $[10,12,16]$. However, as all light from non-structured samples is blocked, it was not possible to reference-correct the reflection spectra as normally done in scatterometry $[10,12,16]$. Instead the spectral properties of each component were calibrated independently, to determine the polarization dependent transmission and reflection properties. For a line grating to provide polarization conversion, the incident polarization must be angled with respect to the grating vector, with an angle of $45^{\circ}$ providing maximum conversion while other angles yield identical spectra but with lower efficiencies [20,21]. The combination of no focus plane, a larger field of view of $5 \times 7 \mathrm{~mm}^{2}$, and rotational independent colors, makes color scatterometry very stable with respect to vibrations and sample misalignments.

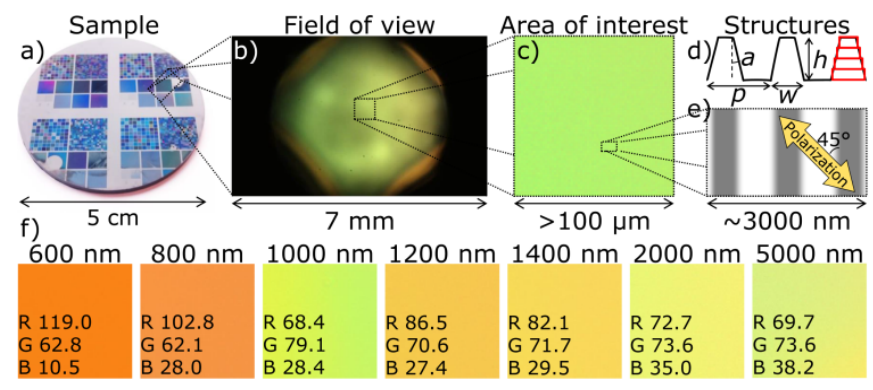

Figure 1: Color scatterometry. a) Photograph of a nano-textured polymer disc. b) Typical color image acquired by the setup, showing one sample area. c) Region cropped from b) and corrected for light non-uniformities by scaling with the RGB intensity. d) Sketch of grating profile, with red boxes illustrating the RCWA simulation setup. e) Illustration of the orientation between line grating and incident polarization. f) Color images of each sample and the corresponding average RGB values. The side lengths are $1 \mathrm{~mm}$.

Theoretical reflection spectra were simulated by rigorous coupledwave analysis (RCWA), which is a computationally efficient technique for calculating reflection efficiencies of periodic structures [10]. In RCWA, the structures are defined as horizontal slabs, for which Maxwell's equations are solved with appropriate boundary conditions between each slab to obtain the grating diffraction efficiencies, see Figure 1d [10,22]. For each sample a range of reflection spectra for different grating dimensions were simulated, and a database of corresponding camera RGB values calculated from Eq (1). By fitting the measured RGB values to the database, the grating dimensions were determined from the best matching simulation, defined by a minimum chi-square value. The $95 \% \mathrm{CI}$ on the dimensions were obtained from constant chi-square boundaries, and the final dimensional value obtained as the midpoint of the CI [10,23].

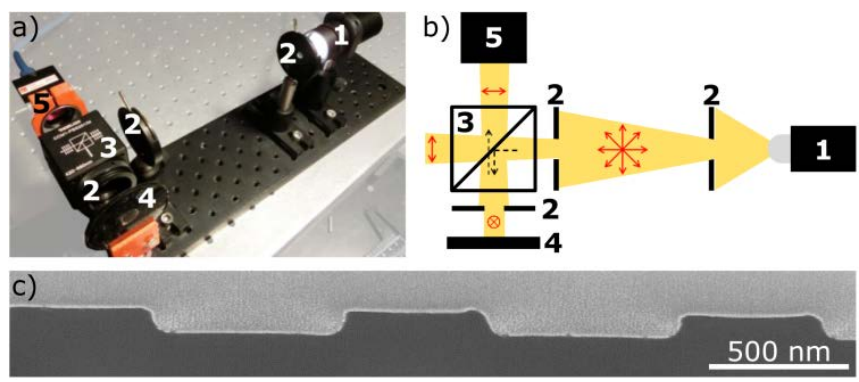

Figure 2: Experimental setup. a) Photograph of the setup, highlighted by a faded background. b) Sketch of setup with the main polarization orientations shown in red. c) FIB SEM images of typical grating profile. Numbers in a) and b) indicate: 1: LED lamp, 2: iris, 3: beamsplitter, 4: sample, 5: camera. 
RCWA simulations were performed using Matlab (Matlab R2014b, MathWorks Inc., USA) and GD-Calc (KJ Innovation, USA). For each grating with a specific height, filling factor, and sidewall slope, the reflection spectra were simulated for wavelengths from $350 \mathrm{~nm}$ to 750 $\mathrm{nm}$ with $5 \mathrm{~nm}$ intervals. The output from a simulation with a single wavelength was a $2 \times 2$ matrix with the reflection efficiencies for $s$ - and $p$-polarized light. The four efficiency values describe both the reflection of unchanged polarization and polarization conversion, with the notation: $r_{s \rightarrow p}$, meaning incident $s$-polarization that is reflected as $p$ polarization. The cross-polarization terms are especially important for the presented setup with crossed polarizers; however, the efficiencies are typically 100 times lower than for the reflection of unchanged polarization. The values were simulated for wavelengths from $350 \mathrm{~nm}$ to $750 \mathrm{~nm}$ with $5 \mathrm{~nm}$ intervals, and the resulting color calculated as:

$$
\begin{gathered}
R=\sum_{i=1}^{N} I_{\mathrm{lamp}}\left(\lambda_{i}\right)\left[\begin{array}{l}
R_{\mathrm{BS}, s}\left(\lambda_{i}\right) \\
R_{\mathrm{BS}, p}\left(\lambda_{i}\right)
\end{array}\right] . \\
{\left[\begin{array}{ll}
r_{s \rightarrow s}\left(\lambda_{i}\right) & r_{s \rightarrow p}\left(\lambda_{i}\right) \\
r_{p \rightarrow s}\left(\lambda_{i}\right) & r_{p \rightarrow p}\left(\lambda_{i}\right)
\end{array}\right]\left[\begin{array}{l}
T_{\mathrm{BS}, s}\left(\lambda_{i}\right) \\
T_{\mathrm{BS}, p}\left(\lambda_{i}\right)
\end{array}\right] S_{\mathrm{cam}, R}\left(\lambda_{i}\right) \Delta \lambda_{i},}
\end{gathered}
$$

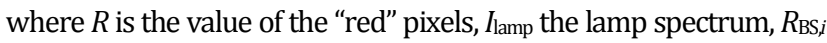
and $T_{\mathrm{BS}, i}$ the beamsplitter reflectance and transmittance for each polarization, respectively, $r_{i \rightarrow j}$ the polarization dependent reflection efficiencies of the sample, $S_{\text {cam, } R}$ the camera spectral sensitivity for channel $R, \Delta \lambda$ the wavelength interval, and $N$ the number of wavelengths. Similar equations were used for the green $(G)$ and blue (B) camera channels.

A typical RGB database consisted of 20.000 different gratings geometries, and took around 15 hours to simulate on a 20-core computer (IBM NeXtScale nx360 M4, Lenovo Group Inc., China), however, the look-up time when fitting to the database requires less with AFM. To provide better resolution of the fitted dimensions without requiring excessive computational time, the resolution of the than one second. The dimensional ranges varied slightly for each sample, but were similar to the one shown in Figure 3b. The ranges were mainly limited due to the computational resource, but were also chosen not to restrict the fitted values. Only except is the height that was limited to the height of the mold-master, determined to $224 \mathrm{~nm}$ simulations were interpolated with a factor of four, resulting in libraries of around 60.000 geometries.

\section{RESULTS AND DISCUSSION}

The reference values obtained by AFM and FIB SEM are shown in Figure 3a. All samples have similar heights and projected area filling factors, indicating good mold filling during fabrication. Only the 600 nm sample deviates with slightly lower values, which is attributed to micro loading effects in the silicon etch process [24]. Three samples $(1000,2000$, and $5000 \mathrm{~nm})$ have large CIs on the slope values, due to demolding damages in the injection molding process, caused by thermal contraction of the polymer that results in scratched sidewalls [25]. Due to spatial variations of the damages, the CIs on the reference values indicate the variation of the grating profile across the sample, and not the accuracy of the reference instrument. Since scatterometry evaluates a mean value covering a larger area, the determined values must overlap the reference range.

The dimensions determined by color scatterometry are compared to the reference values in Figure 3a, and a good correlation for all three dimensions is seen. The color is obtained as the average color of a $1 \times 1$ $\mathrm{mm}^{2}$ region for each sample, see Figure $1 \mathrm{bcf}$. Only the $600 \mathrm{~nm}$ sample deviates in the filling factor. The height and filling factor are determined with average precisions of $7 \%$ and $9 \%$, respectively, corresponding to $14 \mathrm{~nm}$ in the height and 3.5 percentage points in the filling factor. Compared to imaging scatterometry with corresponding values of $3.4 \%$ and $3.0 \%$, the CIs are only two to three times larger [16]. Hence, when considering the significantly shorter measurement time for the presented method, the decrease in precision should not be prohibitive for quality control applications. 

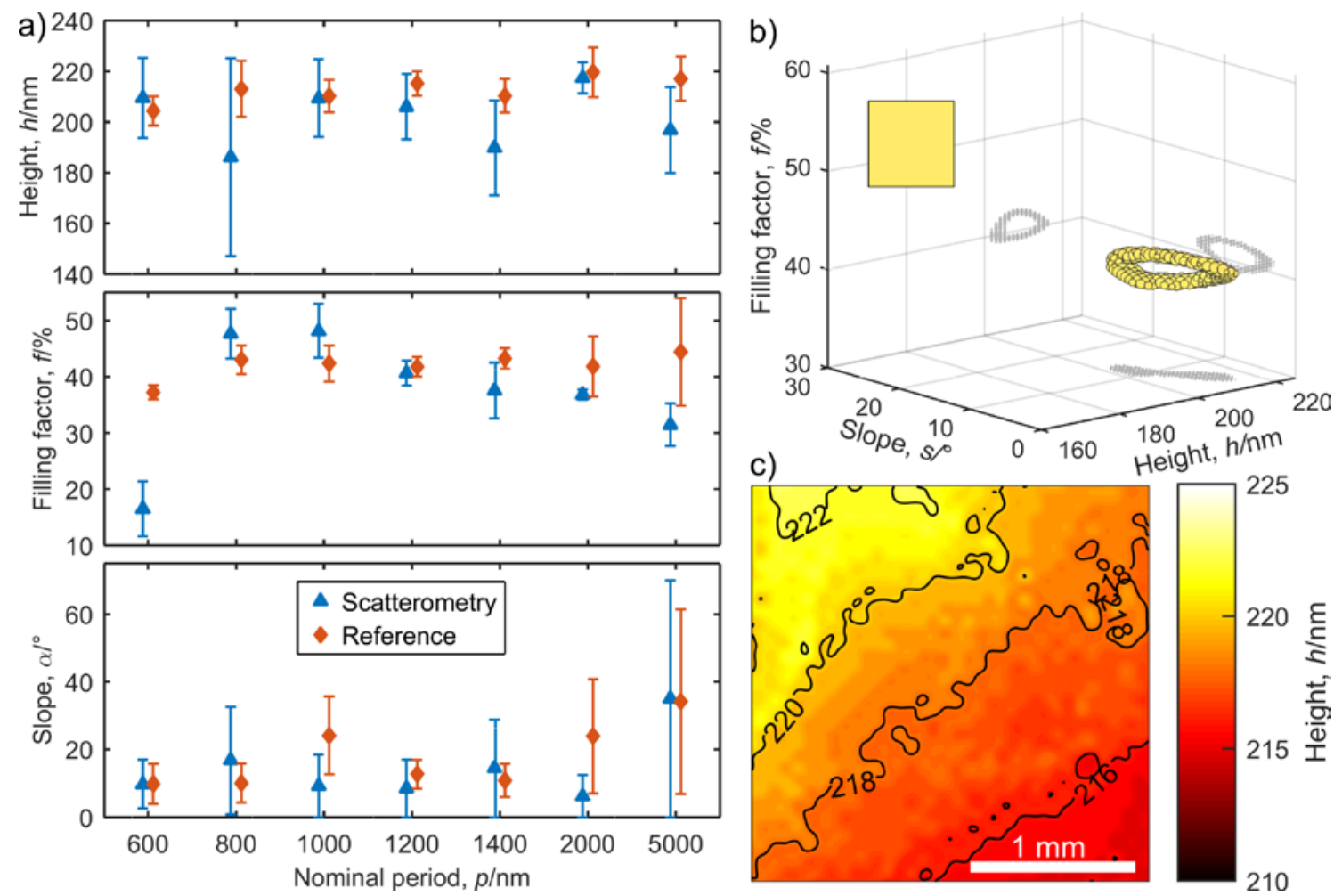

Figure 3: Experimental data. a) Grating dimensions determined for each sample by color scatterometry, and the corresponding reference values obtained with AFM and FIB SEM. Error bars indicate $95 \%$ CIs. b) Illustration of raw fitting output for the $1200 \mathrm{~nm}$ sample. Each dot corresponds to a match between simulated and measured color, within the $95 \%$ CI. Insert shows the measured color, while the markers are colored with the simulated RGB values. c) Contour plot of the fitted height variation over a $6 \mathrm{~mm}^{2}$ sample area, obtained by dividing the image into 1300 individual regions.

The slope, however, is not as well defined, with CIs typically ranging from $0^{\circ}$ to $30^{\circ}$. This is a fundamental limitation of scatterometry being a volume sensitive technique, where the slope only slightly affects the spectrum [16]. The $600 \mathrm{~nm}$ sample matches the reference height and slope, but deviates significantly in the filling factor. The bad fit is also indicated by a significantly larger chi-square value of 0.2 , compared to typically $\sim 0.01$ for the other samples. This single deviation is attributed to the small grating period, resulting in smooth spectra with small color variations. The period is an important parameter for lightstructure interactions, and it cannot be expected that a given scatterometry technique covers all $[10,26]$.

The raw fitting is illustrated as a 3D graph in Figure 3b. The database covers the dimensional ranges shown by the $x, y, z$-axis, in total 15.500 different grating geometries. Each dot indicates a match between simulation and measurement, and the CIs in Figure 3a are determined as the smallest and largest value in each dimension. The marker color corresponds to the simulated color, while the insert shows the sample color. The gray dots illustrate plane projections of the 3D shape. To provide an unambiguous value for the dimensions, only a single region should provide a good fit, similar to the fit shown in Figure $3 \mathrm{~b}$. To test the robustness of the method in terms of this, the $1200 \mathrm{~nm}$ sample was simulated with the dimensional ranges extended down to a height of $140 \mathrm{~nm}$, and a filling factor of only $10 \%$, resulting in a doubling of the number of simulations. Even with this large range of dimensions, the fitting method continued to provide a single region, with the same dimensions as shown in Figure 3a.For some samples, a slight color change is seen across the surface, see Figure 1f. These longranged variations can be analyzed by dividing the image into small independent regions, instead of obtaining a single average value as done for Figure 3a. Such analysis is shown in Figure 3c, where a sample area of $2.5 \mathrm{~mm} \times 2.5 \mathrm{~mm}$ is divided into regions of $10 \times 10$ pixels, and the color of each fitted independently to the RGB database. The analysis reveals a generally increasing height going from the upper left towards the lower right, hereby illustrating the possibilities of analyzing millimeter-sized areas with nanometer precision. The smallest possible crop region is $2 \times 2$ pixels, corresponding to a physical size of $11 \mu \mathrm{m} \times 11 \mu \mathrm{m}$. However, the actual imaging resolution is currently limited to $100 \mu \mathrm{m}$ due to the collimation of the light. The resolution can easily be increased by applying a better collimation, or alternatively, by including a focusing lens.

Generally, the color scatterometry and reference precisions are similar, as illustrated by the confidence intervals in Figure 3a. However, a few samples deviate with much larger or smaller confidence intervals, e.g. the height for $800 \mathrm{~nm}$ and the filling factor for $2000 \mathrm{~nm}$. The precision depends mainly on the gradient of color change, where slowly varying colors result in a low precision due to redundancy effects, where a wide range of e.g. heights is equally probable, while on the contrary very, a drastic color changes yield a higher precision. The color gradient varies very irregularly with the grating dimensions, and can form strange shapes as seen from Figure $3 b$. Hence, to increase the precision in quality control applications, the control structures should be designed with dimension in high gradient regimes. The structures can also be designed to avoid duplicate colors within the range of possible dimensional values, since with the camera acquiring 12-bit RGB values; 69 billion different colors can be distinguished. 


\section{CONCLUSION}

In conclusion, we have presented a new method for optical characterization of nanoscale gratings, which we call "color scatterometry". Color scatterometry is based on spectrographic scatterometry, but instead of obtaining a broad wavelength spectrum, it only evaluates the visual color of the sample, obtained with a single exposure by a RGB color camera. It can evaluate an area of several $\mathrm{mm}^{2}$ in less than a second, and determine the grating dimension with nanometer precision. The height and filling factor are typically obtained with an accuracy of $\pm 8 \%(95 \% \mathrm{Cl})$, while the sidewall slope is within $\pm 15^{\circ}$. The method is tested on polymer samples with nonperfect grating profiles; hence, the presented confidence intervals should reflect realistic values obtainable in actual production environments. So far, color scatterometry has only been validated for line gratings, but adaption to 2D gratings should be straightforward since these structures also influence the specular reflected spectrum [16].

Funding Information. The Danish Agency for Science, Technology and Innovation (Industrial $\mathrm{PhD}$ Programme and the Advanced Technology Group (GTS)).

\section{References}

1. J. S. Clausen, E. Højlund-Nielsen, A. B. Christiansen, S. Yazdi, M. Grajower, H. Taha, U. Levy, A. Kristensen, and N. A. Mortensen, "Plasmonic metasurfaces for coloration of plastic consumer products," Nano Lett. 14, 4499-4504 (2014).

2. X. M. Goh, Y. Zheng, S. J. Tan, L. Zhang, K. Kumar, C.-W. Qiu, and J. K. W. Yang, "Three-dimensional plasmonic stereoscopic prints in full colour," Nat. Commun. 5, 5361 (2014).

3. P. Roach, N. J. Shirtcliffe, and M. I. Newton, "Progess in superhydrophobic surface development," Soft Matter 4, 224-240 (2008)

4. E. Søgaard, N. K. Andersen, K. Smistrup, S. T. Larsen, L. Sun, and R. Taboryski, "Study of Transitions between Wetting States on Microcavity Arrays by Optical Transmission Microscopy," Langmuir 30, 1296012968 (2014)

5. M. Zalkovskij, L. H. Thamdrup, K. Smistrup, T. Andén, A. C. Johansson, N. J. Mikkelsen, M. H. Madsen, J. Garnæs, T. T. Kristiansen, M. Diemer, M. Døssing, D. Minzari, P. T. Tang, A. Kristensen, R. Taboryski, S. Essendrop, T. Nielsen, and B. Bilenberg, "Smart plastic functionalization by nanoimprint and injection molding," in Proc. SPIE 9423, Alternative Lithographic Technologies VII, D. J. Resnick and C. Bencher, eds. (2015), Vol. 9423, p. 94230T.

6. S. Murthy, M. Matschuk, Q. Huang, N. K. Mandsberg, N. A. Feidenhans'I, P. Johansen, L. Christensen, H. Pranov, G. Kofod, H. C. Pedersen, O. Hassager, and R. Taboryski, "Fabrication of Nanostructures by Roll-toRoll Extrusion Coating," Adv. Eng. Mater. 18, 484-489 (2016).

7. S. H. Ahn and L. J. Guo, "High-Speed Roll-to-Roll Nanoimprint Lithography on Flexible Plastic Substrates," Adv. Mater. 20, 2044-2049 (2008)

8. S. H. Ahn and L. J. Guo, "Large-Area Roll-to-Roll and Roll-to-Plate Nanoimprint Lithography: A Step toward High-Throughput Application of Continuous Nanoimprinting," ACS Nano 3, 2304-2310 (2009).

9. M. Leitgeb, D. Nees, S. Ruttloff, U. Palfinger, J. Götz, R. Liska, M. R. Belegratis, and B. Stadlober, "Multilength Scale Patterning of Functional Layers by Roll-to-Roll Ultraviolet-Light-Assisted Nanoimprint Lithography," ACS Nano 10, 4926-4941 (2016).

10. 1M. H. Madsen and P.-E. Hansen, "Scatterometry-fast and robust measurements of nano-textured surfaces," Surf. Topogr. Metrol. Prop. 4, 023003 (2016).

11. H. H. Smilde, A. den Boef, M. Kubis, M. Jak, M. van Schijndel, A. Fuchs, M. van der Schaar, S. Meyer, S. Morgan, J. Wu, V. Tsai, C. Wang, K.
Bhattacharyya, K.-H. Chen, G.-T. Huang, C.-M. Ke, and J. Huang, "Evaluation of a novel ultra small target technology supporting onproduct overlay measurements," in Proc. SPIE 8324, Metrology, Inspection, and Process Control for Microlithography XXVI, A. Starikov, ed. (2012), Vol. 8324, p. 83241A

12. M. H. Madsen, P.-E. Hansen, M. Zalkovskij, M. Karamehmedović, and J. Garnæs, "Fast characterization of moving samples with nano-textured surfaces," Optica 2, 301-306 (2015).

13. S. Peterhänsel, H. Laamanen, M. Kuittinen, J. Turunen, C. Pruss, W. Osten, and J. Tervo, "Solving the inverse grating problem with the naked eye," Opt. Lett. 39, 3547-50 (2014).

14. S. Peterhänsel, H. Laamanen, J. Lehtolahti, M. Kuittinen, W. Osten, and J. Tervo, "Human color vision provides nanoscale accuracy in thin-film thickness characterization," Optica 2, 627 (2015).

15. J. Henrie, S. Kellis, S. Schultz, and A. Hawkins, "Electronic color charts for dielectric films on silicon.," Opt. Express 12, 1464-9 (2004).

16. M. H. Madsen and P.-E. Hansen, "Imaging scatterometry for flexible measurements of patterned areas," Opt. Express 24, 1109-1117 (2016).

17. N. A. Feidenhans'I, P.-E. Hansen, L. Pilný, M. H. Madsen, G. Bissacco, J. C. Petersen, and R. Taboryski, "Comparison of optical methods for surface roughness characterization," Meas. Sci. Technol. 26, 085208 (2015)

18. J. C. Stover, Optical Scattering: Measurement and Analysis, 3. Edition (SPIE Press, 2012)

19. W. G. Cochran, "The distribution of quadratic forms in a normal system, with applications to the analysis of covariance," Math. Proc. Cambridge Philos. Soc. 30, 178 (1934).

20. J. B. Harris, T. W. Preist, E. L. Wood, and J. R. Sambles, "Conical diffraction from multicoated gratings containing uniaxial materials," J. Opt. Soc. Am. A 13, 803 (1996).

21. G. P. Bryan-Brown, S. J. Elston, and J. R. Sambles, "Polarization conversion through the excitation of electromagnetic modes on a grating," in Proc. SPIE 1545, International Conference on the Application and Theory of Periodic Structures (1991), Vol. 1545, pp. 167-178.

22. KJ Innovation, Optical Simulation of Grating Diffraction in MATLAB (GDcalc_Intro.pdf) (2006).

23. W. H. Press, W. T. Vetterling, S. a Teukolsky, and B. P. Flannery, Numerical Recipes in C: The Art of Scientific Computing, 2. ed. (Cambridge University Press, 1992).

24. J. D. Plummer, M. Deal, and P. D. Griffin, Silicon VLSI Technology (Prentice Hall, 2000).

25. G. Fu, S. B. Tor, D. E. Hardt, and N. H. Loh, "Effects of processing parameters on the micro-channels replication in microfluidic devices fabricated by micro injection molding," Microsyst. Technol. 17, 17911798 (2011)

26. P. E. Hansen and L. Nielsen, "Combined optimization and hybrid scalarvector diffraction method for grating topography parameters determination," Mater. Sci. Eng. B Solid-State Mater. Adv. Technol. 165, 165-168 (2009). 